\title{
Computing the Maximum Overlap of Two Convex Polygons Under Translations*
}

\author{
Mark de Berg $^{1} \quad$ Otfried Cheong $^{2} \quad$ Olivier Devillers $^{3} \quad$ Marc van Kreveld $^{1}$ \\ Monique Teillaud ${ }^{3}$
}

\begin{abstract}
Let $P$ be a convex polygon in the plane with $n$ vertices and let $Q$ be a convex polygon with $m$ vertices. We prove that the maximum number of combinatorially distinct placements of $Q$ with respect to $P$ under translations is $O\left(n^{2}+m^{2}+\min \left(n m^{2}+n^{2} m\right)\right)$, and we give an example showing that this bound is tight in the worst case. Second, we present an $O((n+m) \log (n+m))$ algorithm for determining a translation of $Q$ that maximizes the area of overlap of $P$ and $Q$.

We also prove that the placement of $Q$ that makes the centroids of $Q$ and $P$ coincide realizes an overlap of at least $9 / 25$ of the maximum possible overlap. As an upper bound, we show an example where the overlap in this placement is $4 / 9$ of the maximum possible overlap.
\end{abstract}

\section{Introduction}

Matching plays an important role in areas such as computer vision. Typically one is given two 'shapes' - point sets or polygons, for instance - and one wants to determine how much these shapes resemble each other. More precisely, one wants to find a rigid motion of one shape that maximizes the resemblance with the other shape. There are several ways to measure resemblance. For example, for point sets or polygonal chains one can use the Hausdorff distance [2, 1, 9, 15, 16]; for polygonal chains one can also use the Fréchet distance [3].

The resemblance of two convex polygons can also be measured by looking at the Hausdorff or Fréchet distance between their boundaries. For an application in computer vision, however, it seems more appropriate to look at the area of the symmetric difference of the two polygons, since this distance measure is less sensitive to noise in the image: noise may add thin features to the boundary but is unlikely to add large areas.

Notice that minimizing the area of the symmetric difference of two polygons is equivalent to maximizing the area of overlap of the polygons. An algorithm with $O(n(n+m))$ time complexity is known for finding the maximum overlap area for two convex polygons, one of which is allowed to rotate with one point on its boundary sliding on the other polygon's boundary [21]. Mount et al. [18] studied the behavior of the area of overlap for two simple polygons under translations of one polygon. They pose the case of two convex polygons as an open problem.

We consider the matching problem for convex polygons in the plane, and the rigid motions that we allow are translations. In other words, we are given two convex polygons $P$ and $Q$ in the plane, and our goal is to find a translation of $Q$ that maximizes the area of overlap with $P$. Our

\footnotetext{
${ }^{*}$ This work was supported by ESPRIT Basic Research Action No. 7141 (project ALCOM II: Algorithms and Complexity). M.d.B. and O.C were also supported by the Netherlands' Organisation for Scientific Research (NWO). O.C. acknowledges partial support by the nondirected research grant of the Korean Ministry of Education.

${ }^{1}$ Dept. of Computer Science, Utrecht University, P.O. Box 80.089, 3508 TB Utrecht, the Netherlands.

${ }^{2}$ Dept. of Computer Science, Hong Kong University of Science and Technology, Clear Water Bay, Kowloon, Hong Kong.

${ }^{3}$ INRIA, BP93, 06902 Sophia-Antipolis cedex.
} 
results are as follows. Let $n$ and $m$ denote the number of vertices of $P$ and $Q$, respectively. We start by studying a combinatorial question: how many combinatorially distinct placements of $Q$ with respect to $P$ are there? Here we define two placements to be combinatorially equivalent if the same pairs of edges (one from $P$ and one from $Q$ ) intersect-see Section 2 for a more precise definition. We show that the number of distinct placements is $O\left(n^{2}+m^{2}+\min \left(n m^{2}+n^{2} m\right)\right)$, and we give an example showing that this bound is tight in the worst case. To our surprise, this result appears to be new: previous work on bounding the number of placements of a polygon in a polygonal environment is usually motivated by motion planning problem and, hence, only deals with the case where the polygon is not allowed to intersect the environment at all - see Latombe's book [17] or Halperin's thesis [14]. Our main result is presented in Section 3, where we give an $O((n+m) \log (n+m))$ time algorithm for computing a placement of $Q$ that maximizes the area of overlap with $P$. Our algorithm is based on the fact that the area-of-overlap function is unimodal. To round off our exposition, we show that one can, in a sense, approximate the maximum possible overlap of two convex polygons by simply superposing them such that their centroids coincide. We show that that placement realizes an overlap that is at least $9 / 25$ of the maximum possible overlap, and we give an upper bound example where the factor is $4 / 9$.

Our work can also be seen as a generalization of the problem of placing a copy of one polygon inside another polygon. Chazelle [6] studied several variants of this problem. One of his results is that, given two convex polygons $P$ and $Q$, one can decide in linear time whether $Q$ can be translated such that it is contained in $P$. Other papers compute the largest copy of a polygon that can be placed inside another one [5, 10, 11, 20].

\section{The number of distinct placements}

Let $P$ be a simple polygon with $n$ vertices in the plane and let $Q$ be a simple polygon with $m$ vertices. The position and orientation of $P$ are fixed, but $Q$ is free to translate. In this section we bound the number of distinct placements of $Q$ with respect to $P$. We first define formally when we call two placements distinct.

We denote the boundary of $P$ by $\partial P$, and the boundary of $Q$ by $\partial Q$. We consider boundary edges to be relatively open sets, that is, their endpoints are not included. Let $r_{Q}$ be a reference point on $Q$, say the lexicographically smallest vertex. For a point $r$ in the plane, $Q(r)$ denotes $Q$ with its reference point placed at $r$. Similarly, for an edge $e$ or a vertex $v$ of $Q, e(r)$ and $v(r)$ denote the edge $e$ and vertex $v$ when $Q$ is placed at $r$. We call $Q(r)$ a placement of $Q$. The space of all possible placements of $Q$-in our case this is a 2-dimensional space-is called the configuration space [17].

Definition 2.1 The intersection set of $P$ and a placement $Q(r)$, denoted $I(r)$, is the set consisting of all pairs $(f, g)$ such that $f$ is the interior of $P$, an edge of $P$, or a vertex of $P, g$ is the interior of $Q(r)$, an edge of $Q(r)$, or a vertex of $Q(r)$, and $f$ and $g$ intersect. Two placements $Q(r)$ and $Q\left(r^{\prime}\right)$ are combinatorially distinct if and only if $I(r) \neq I\left(r^{\prime}\right)$.

The configuration space can be partitioned into regions according to the intersection sets of the corresponding placements: two points are in the same region if and only if the corresponding placements are combinatorially equivalent. Hence, the number of combinatorially distinct placements is bounded by the number of regions in the configuration space.

Previous work on configuration spaces was usually inspired by motion planning applications, where the polygon $Q$ is a robot, the polygon $P$ is an obstacle. In this setting, one is interested in the free space, the region of the configuration space where $Q$ does not collide with $P$ or, in other words, where the intersection set is empty. When only translations are considered, then the free space is the complement of the Minkowski sum of $P$ and $-Q$, and its complexity is $\Theta\left(n^{2} m^{2}\right)$ in the worst case; for convex polygons, the complexity is $\Theta(n+m)$ in the worst case. For more information and references on configuration spaces in connection with motion planning we refer the 
reader to Latombe's book [17] or Halperin's thesis [14]. In our application we are also interested in placements where the intersection set is not empty, so few results from the motion planning literature seem to apply [19].

Let's have a closer look at the configuration space. Fix an edge $e$ of $P$ and an edge $e^{\prime}$ of $Q$, and consider the locus of all points $r$ such that $e$ intersects $e^{\prime}(r)$. This region is a parallelogram, denoted $\pi\left(e, e^{\prime}\right)$, spanned by a translated copy of $e$ and a translated copy of $e^{\prime}$. Observe that for points $r$ in the interior of the edges of $\pi\left(e, e^{\prime}\right)$, a vertex of $e$ lies on $e^{\prime}(r)$ or a vertex of $e^{\prime}(r)$ lies on $e$; for a point $r$ that is a vertex of $\pi\left(e, e^{\prime}\right)$, a vertex of $e$ coincides with a vertex of $e^{\prime}(r)$. Let

$$
\Pi=\left\{\pi\left(e, e^{\prime}\right): e \text { is an edge of } P, e^{\prime} \text { is an edge of } Q\right\} .
$$

The arrangement $\mathcal{A}(\Pi)$ induced by $\Pi$ is the partitioning of configuration space we mentioned above: there is a one-to-one correspondence between the combinatorially distinct placements and the faces, arcs, ${ }^{1}$ and nodes of $\mathcal{A}(\Pi)$. So a bound on the complexity of $\mathcal{A}(\Pi)$ immediately implies a bound on the number of distinct placements.

We proceed to bound the complexity of $\mathcal{A}(\Pi)$. Because $\mathcal{A}(\Pi)$ is a planar subdivision defined by $n m$ parallelograms, its complexity is bounded by $O\left(n^{2} m^{2}\right)$. For simple polygons, this bound is tight in the worst case. In fact, the complexity of the free space can already be $\Theta\left(n^{2} m^{2}\right)$, as mentioned above.

For convex polygons, the situation is different: We show in the following theorem that the maximum number of combinatorially distinct placements is $\Theta\left(n^{2}+m^{2}+\min \left(n m^{2}, n^{2} m\right)\right)$. While this is significantly less than $\Theta\left(n^{2} m^{2}\right)$, it is much larger than the complexity of the free space for two convex polygons.

Theorem 2.2 The maximum number of combinatorially distinct placements of two convex polygons with $n$ and $m$ vertices, respectively, is:

$$
\Theta\left(n^{2}+m^{2}+\min \left(n m^{2}, n^{2} m\right)\right) .
$$

Proof: We first prove the upper bound. Let $P$ be a convex polygon with $n$ vertices, and $Q$ a convex polygon with $m$ vertices. We bound the complexity of the subdivision $\mathcal{A}(\Pi)$ we get for $P$ and $Q$, as defined above. Because $\mathcal{A}(\Pi)$ is a planar subdivision, it suffices to bound its number of nodes. A node of $\mathcal{A}(\Pi)$ is either a corner of some parallelogram $\pi\left(e, e^{\prime}\right)$ or an intersection between the boundary of two such parallelograms. The corners of the parallelograms corresponds to a placement where a vertex of $Q$ coincides with a vertex of $P$; clearly there are $O(n m)$ of these placements. The intersections between parallelogram boundaries correspond to placements such that

i. there are edges $e_{1}, e_{2}$ of $P$ and vertices $v_{1}, v_{2}$ of $Q$ such that $v_{i} \in e_{i}$, for $i=1,2$, or

ii. there are vertices $v_{1}, v_{2}$ of $P$ and edges $e_{1}, e_{2}$ of $Q$ such that $v_{i} \in e_{i}$, for $i=1,2$, or

iii. there is an edge $e_{1}$ of $P$, a vertex $v_{2}$ of $P$, a vertex $v_{1}$ of $Q$ and an edge $e_{2}$ of $Q$ such that $v_{i} \in e_{i}$, for $i=1,2$.

First we bound the number of nodes of type (i). Fix one vertex $v_{1}$ of $Q$, and place $v_{1}$ somewhere on $\partial P$. Now move $Q$ 'around' $P$, while keeping $v_{1}$ on $\partial P$. We get a type (i) node when a vertex of $Q$ crosses a edge of $P$. Because the path that every vertex of $Q$ describes is a translate of $\partial P$, it can intersect $\partial P$ at most twice. Hence, the total number of type (i) nodes involving vertex $v_{1}$ is at most $2 \mathrm{~m}$. The total number of type (i) nodes over all vertices of $Q$ is therefore $O\left(\mathrm{~m}^{2}\right)$.

A similar argument shows that the number of type (ii) nodes is $O\left(n^{2}\right)$.

\footnotetext{
${ }^{1}$ To avoid confusion between the edges of the polygons and the edges of $\mathcal{A}(\Pi)$, we call the latter arcs. Similarly, we call the vertices of $\mathcal{A}(\Pi)$ nodes.
} 
It remains to bound the number of nodes of type (iii). We fix a vertex $v_{2}$ of $P$ and move $Q$ 'around' $P$ while $v_{2}$ stays on $\partial Q$. We must count the number of times that a vertex of $Q$ crosses an edge of $P$. Let's look at the path that a vertex $v_{1}$ of $Q$ follows. This path can be obtained by placing $v_{1}$ at $v_{2}$ and rotating $Q$ over 180 degrees around $v_{1}$; the mirrored image of $Q$ that results is exactly the path that $v_{1}$ follows. So the path is convex and polygonal, and it has $m$ segments. Hence, $v_{1}$ crosses $\partial P$ at most $O(\min (n, m))$ times. The number of type (iii) nodes involving vertex $v_{2}$ of $P$ is therefore $O(m \min (n, m))$, and the total number of type (iii) nodes is $O(n m \min (n, m))$.

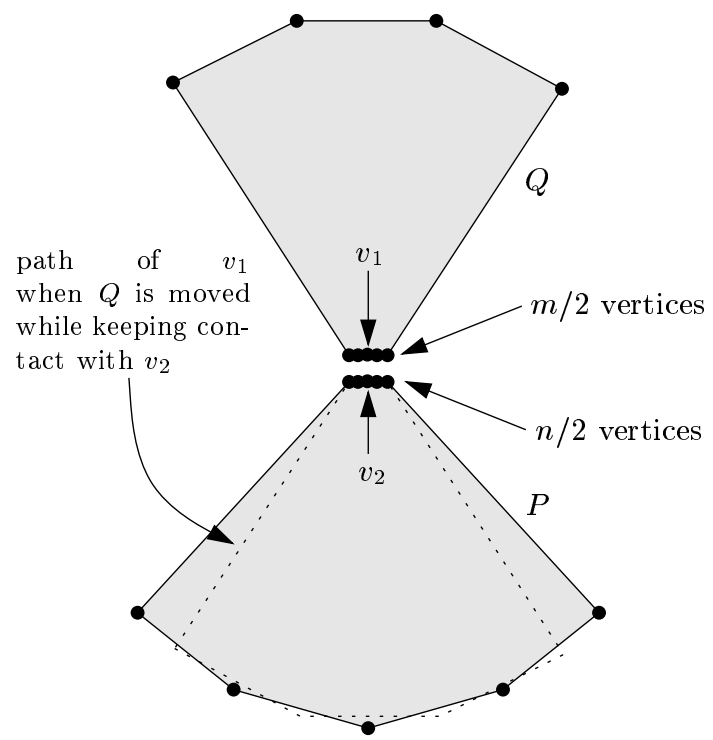

Figure 1: Two convex polygons with $\Omega\left(\min \left(n m^{2}+n^{2} m\right)\right)$ distinct placements.

An example where there are $\Omega\left(n^{2}+m^{2}\right)$ distinct placements is easy to construct, so we only give an example with $\Omega(n m \min (n, m))$ distinct placements. Fig. 1 gives such an example. The dotted polygonal closed path is the path $v_{1}$ follows when $Q$ is moved while keeping contact with $v_{2}$. This path intersects $\partial P \Omega(\min (n, m))$ times. Let $w_{1}$ be any of the $m / 2$ bottom vertices of $Q$ and $w_{2}$ any of the $n / 2$ top vertices of $P$. When the top vertices of $P$ and the bottom vertices of $Q$ are placed close enough together, then the path followed by $w_{1}$ when $Q$ is moved around $w_{2}$ will be close enough to the dotted path, so that there will be $\Omega(\min (n, m))$ intersections of the path with $\partial P$. Hence, we get a total of $\Omega(n m \min (n, m))$ distinct placements.

\section{Computing the maximum overlap}

We now get to the main problem studied in this paper: given two convex polygons $P$ and $Q$, find a placement of $Q$ that maximizes the overlap with $P$. First, we need to introduce some notation. The overlap function $\omega(r): \mathbb{R}^{2} \rightarrow \mathbb{R}$ of $P$ and $Q$ is defined as

$$
\omega(r):=\text { the area of } P \cap Q(r) .
$$

Our problem is thus to find a placement $Q(r)$ that maximizes $\omega(r)$. We call such a placement a goal placement. 
We first look at a restricted version of the problem, where $Q$ is only allowed to translated into a fixed direction. Without loss of generality, we assume this direction to be horizontal. Thus, for a given value $y^{*}$, we define the (horizontal) overlap function at $y^{*}$, denoted by $\omega_{y^{*}}(t)$, as

$$
\omega_{y^{*}}(t):=\omega\left(\left(t, y^{*}\right)\right)
$$

Theorem 3.1 Let $P$ and $Q$ be two convex polygons then the function $r \mapsto \sqrt{\omega(r)}$ is downwards concave, that is the volume below its graph is convex.

Proof: Since a function $\mathbb{R}^{2} \rightarrow \mathbb{R}$ is downwards concave if any cross-section along a line is downwards concave, it suffices to prove the latter fact. Without loss of generality, we can restrict ourself to the case of horizontal lines, and will prove that the monovariate function $t \mapsto \sqrt{\omega_{y^{*}}(t)}$ is downwards concave.

Imagine moving $Q$ from left to right over the plane, starting with $Q\left(\left(-\infty, y^{*}\right)\right)$ and ending at $Q\left(\left(+\infty, y^{*}\right)\right)$. Define $Q(t):=Q\left(\left(t, y^{*}\right)\right)$, and $A(t):=P \cap Q(t)$. Thus $A(t)$ is the intersection of $P$ and $Q$ at time $t$. We define a three-dimensional polytope $\mathcal{P}_{P Q}$ by viewing time as the third dimension, and taking the union of all polygons $A(t)$ :

$$
\mathcal{P}_{P Q}:=\{(x, y, t):(x, y) \in A(t)\} .
$$

Since $\mathcal{P}_{P Q}$ can be written as the intersection of two convex polytopes, it is a convex polytope itself:

$$
\mathcal{P}_{P Q}=\{(x, y, t):(x, y) \in P\} \cap\{(x, y, t):(x, y) \in Q(t)\}
$$

Following Avis et al. [4], we can now apply the Brunn-Minkowski theorem [13], which states that the square root of the function that describes the area of intersection of $\mathcal{P}_{P Q}$ and a horizontal plane $h$ is downwards concave, as we sweep $h$ through $\mathcal{P}_{P Q}$. Since the cross-section of $\mathcal{P}_{P Q}$ with the horizontal plane $t=t^{*}$ is exactly the intersection $A\left(t^{*}\right)$, the theorem follows.

A non-negative function $\chi: D \rightarrow \mathbb{R}$ is called unimodal if there is an interval $D=\left[a_{0}: a_{1}\right]$ and points $b_{0}, b_{1} \in D$ with $b_{0} \leqslant b_{1}$ such that $\chi$ is zero outside $D$, strictly increasing from $a_{0}$ to $b_{0}$, constant from $b_{0}$ to $b_{1}$, and strictly decreasing from $b_{1}$ to $a_{1}$. Our algorithm is based on the unimodality of the monovariate overlap function.

Corollary 3.2 Let $P$ and $Q$ be two convex polygons, and let $y^{*} \in \mathbb{R}$. Then the horizontal overlap function $\omega_{y^{*}}(t)$ is unimodal.

Proof: From the downwards concavity of $\sqrt{\omega_{y^{*}(t)}}$ immediately follows that it is a unimodal function, which in turn implies that $\omega_{y^{*}}(t)$ is unimodal as well.

Theorem 3.2 can be used to compute the maximum overlap of $P$ and $Q$ for the case where $Q$ is confined to translate along a fixed line. This algorithm will be an important ingredient of the general algorithm.

Lemma 3.3 [Avis et al.] For a line $\ell$ we can compute $\max _{r \in \ell} \omega(r)$ in $O(n+m)$ time.

Proof: Using Chazelle's algorithm [7] the convex polytope $\mathcal{P}_{P Q}$ can be computed in linear time, and then Avis et al.'s algorithm [4] can be used to compute the horizontal section of $\mathcal{P}_{P Q}$ of maximal area in linear time.

We now turn our attention to the general case, where arbitrary translations are allowed. Our algorithm consists of two stages. In the first stage we locate a horizontal strip that contains the reference point of a goal placement. This will be done by a binary search that uses the algorithm from Lemma 3.3 as a subroutine. This reduces the complexity of the search space sufficiently to enter the second stage of the algorithm, which is based on cuttings. The second stage reduces the complexity of the search space further so that it becomes easy to compute the maximum overlap. We now describe the stages in more detail. 
The first stage. Consider a placement where $Q$ is completely below $P$, and imagine moving $Q$ upward until it is entirely above $P$. Let $Y=y_{1}, y_{2}, \ldots, y_{n m}$ be the sorted sequence of $y$-values where a vertex of $Q$ and a vertex of $P$ align horizontally. In other words, $Y$ contains the values $y_{i}$ such that there are vertices $v$ of $P$ and $w$ of $Q\left(\left(x, y_{i}\right)\right)$ with the same $y$-coordinate. We shall do a binary search on $Y$ to locate a horizontal strip $[-\infty: \infty] \times\left[y_{i}: y_{i+1}\right]$ that contains a goal placement. (In fact, we should write 'that contains the reference point of a goal placement'. When no confusion can arise, we shall permit ourselves this slight abuse of terminology.) We do not compute the set $Y$ explicitly, however, because $Y$ can contain $n m$ elements and we do not want to spend that much time.

Let's look more closely at the set $Y$. Let $A=\left\{a_{1}, \ldots, a_{n}\right\}$ be the set of $y$-coordinates of the vertices of $P$, sorted in increasing order, and let $B=\left\{b_{1}, \ldots, b_{n}\right\}$ be the set of $y$-coordinates of the vertices of $Q((0,0))$, sorted in decreasing order. The sets $A$ and $B$ can be computed in linear time. The elements of the set $Y$ are exactly the entries of the matrix

$$
\mathcal{M}=\left(c_{i j}\right), \text { where } c_{i j}=a_{i}-b_{j} .
$$

Because the sets $A$ and $B$ are sorted, every row and every column of $\mathcal{M}$ is sorted. Furthermore, an entry $c_{i j}$ can be evaluated in constant time. Hence, for any parameter $k$ with $1 \leqslant k \leqslant n m$, we can compute the $k$-th largest entry of $\mathcal{M}$ in $O(m \log (2 n / m)) \leqslant O(n+m)$ time with an algorithm by Frederickson and Johnson [12].

The binary search now proceeds as follows. In a generic step we have two values, $k_{\min }$ and $k_{\max }$ such that there is a goal placement in the horizontal strip $[-\infty: \infty] \times\left[y_{k_{\min }}: y_{k_{\max }}\right]$. Initially $k_{\min }=0$ and $k_{\max }=n m$. We first compute the values $y_{k}$ and $y_{k+1}$, where $k=\left\lfloor\left(k_{\min }+k_{\max }\right) / 2\right\rfloor$, with the algorithm of Frederickson and Johnson. Then we compute $\max _{t} \omega_{y_{k}}(t)$ and $\max _{t} \omega_{y_{k+1}}(t)$ using Lemma 3.3. There are three cases to consider, depending on the computed values:

$$
\begin{aligned}
& \text { If } \max _{t} \omega_{y_{k}}(t)<\max _{t} \omega_{y_{k+1}}(t) \text { then we set } k_{\min }:=k \\
& \text { If } \max _{t} \omega_{y_{k}}(t)>\max _{t} \omega_{y_{k+1}}(t) \text { then we set } k_{\max }:=k+1 \\
& \text { If } \max _{t} \omega_{y_{k}}(t)=\max _{t} \omega_{y_{k+1}}(t) \text { then we set } k_{\min }:=k \text { and } k_{\max }:=k+1 \text { and we have } \\
& \text { found the strip. }
\end{aligned}
$$

The binary search continues until $k_{\max }-k_{\min }=1$. The correctness of the algorithm is based on the following lemma.

Lemma 3.4 Let $\ell_{1}$ and $\ell_{2}$ be two lines, and let $r_{1}$ and $r_{2}$ be points on $\ell_{1}$ and $\ell_{2}$, respectively, such that $\omega\left(r_{1}\right)=\max _{r \in \ell_{1}} \omega(r)$ and $\omega\left(r_{2}\right)=\max _{r \in \ell_{2}} \omega(r)$. If $\omega\left(r_{1}\right) \geqslant \omega\left(r_{2}\right)>0$ and $r_{1}$ does not lie on $\ell_{2}$ then the open half-plane bounded by $\ell_{2}$ and containing $r_{1}$ contains a goal placement.

Proof: We shall prove that the closed half-plane bounded by $\ell_{2}$ and not containing $r_{1}$ cannot

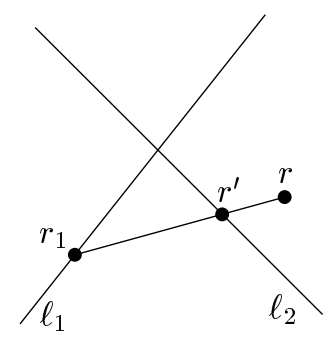

Figure 2: 
contain a placement $r$ such that $\omega(r)>\omega\left(r_{1}\right)$, which implies the lemma. Let $r$ be any point in this half-plane, and let $r^{\prime}$ be the intersection point of the closed line segment $\overline{r r_{1}}$ with $\ell_{2}$. Because the overlap function is unimodal, $\omega(r)>\omega\left(r_{1}\right)$ would imply $\omega\left(r^{\prime}\right)>\omega\left(r_{1}\right)$. But since $\omega\left(r_{2}\right) \geqslant \omega\left(r^{\prime}\right)$ by definition of $r_{2}$, this would contradict the assumption that $\omega\left(r_{1}\right) \geqslant \omega\left(r_{2}\right)$.

We can now prove that the binary search algorithm correctly and efficiently finds a strip containing a goal placement.

Lemma 3.5 The binary search finds in time $O((n+m) \log (n+m))$ a horizontal strip:

$$
[-\infty: \infty] \times\left[y_{i}: y_{i+1}\right]
$$

that contains a goal placement.

Proof: Let's first prove that the algorithm is correct. This amounts to proving that the three cases mentioned above are handled correctly. Let $t_{1}$ be a value maximizing $\omega_{y_{k}}(t)$ and let $t_{2}$ be a value maximizing $\omega_{y_{k+1}}(t)$. Define $r_{1}=\left(t_{1}, y_{k}\right)$ and $r_{2}=\left(t_{2}, y_{k+1}\right)$. Suppose that $\omega\left(r_{1}\right) \leqslant \omega\left(r_{2}\right)$. By Lemma 3.4 there must be a goal placement above the line $y=y_{k}$, which proves that the first case is handled correctly. Similarly, $\omega\left(r_{1}\right) \geqslant \omega\left(r_{2}\right)$ implies that there is a goal placement below the line $y=y_{k+1}$, which proves that the second case is handled correctly. By combining the arguments for the first two cases, we see that also the third case is handled correctly.

It remains to prove the time bound. In each step of the binary search we use the selection algorithm of Frederickson and Johnson [12], which takes $O(m+n)$ time, and we apply the algorithm of Lemma 3.3, which takes $O(n+m)$ time. Since the number of steps of the binary search is $O(\log (n m))$, the total time is as claimed.

The binary search on the set $Y$ gives us a horizontal strip that contains a goal placement. For any placement $Q(r)$ in the interior of this strip, the vertical order of the vertices of $P$ with respect to those of $Q(r)$ is fixed. This means that the complexity of the part of $\mathcal{A}(\Pi)$ within $R$ is significantly less than the total complexity of $\mathcal{A}(\Pi)$, as we show next.

Lemma 3.6 After the first stage of the algorithm we have located a horizontal strip $\sigma=[-\infty$ : $\infty] \times\left[y: y^{\prime}\right]$ containing a goal placement such that the part of $\mathcal{A}(\Pi)$ inside $\sigma$ is formed by $O(n+m)$ segments.

Proof: Recall that $\mathcal{A}(\Pi)$ is defined by $O(n m)$ parallelograms. Each parallelogram is defined by a pair of edges, one from $P$ and one from $Q$. The edges of these parallelograms, in other words, the segments that induce $\mathcal{A}(\Pi)$, are defined by a vertex-edge pair. We claim that a vertex can define at most two vertex-edge pairs whose corresponding segment intersects $\sigma$. Let $v$ be a vertex of $Q$, and let $e$ be an edge of $P$. Let $Q(r)$ be a placement with $r \in \sigma$. If the horizontal line through $v(r)$ does not intersect $e$ then $v$ exchanges its vertical order with an endpoint of $e$ when it is moved to lie on $e$. Hence, $v$ can only define a vertex-edge pair with an edge $e$ intersected by the horizontal line through $v(r)$. Because $P$ is convex there are at most two such edges. The same argument shows that any vertex of $P$ can define at most two vertex-edge pairs.

The second stage We enter the second stage with a horizontal strip $\sigma=[-\infty: \infty] \times\left[y: y^{\prime}\right]$ that contains a goal position. The number of segments defining $\mathcal{A}(\Pi)$ inside $\sigma$ is $O(n+m)$. From the proof of Lemma 3.6 it follows that we can compute these segments in linear time: take a point $r$ inside the strip, and merge the two sorted sequences of $y$-coordinates of the vertices of $P$ and the vertices of $Q(r)$ to find for each vertex the at most two edges with which it can define an edge inside $\sigma$. Because we know the segments defining $\mathcal{A}(\Pi) \cap \sigma$, we can use cuttings to zoom in further on a goal placement. How this works is explained next.

Let $S$ be a set of line segments in the plane. A $(1 / k)$-cutting $\Xi(S)$ for $S$ is a collection of triangles with disjoint interiors that collectively cover the entire plane, such that for each triangle 
in $\Xi(S)$ the number of segments intersecting its interior is at most $|S| / k$. The size of a cutting is the number of simplices it consists of. For any set of lines in the plane-and, hence, for any set of line segments - there is a cutting of size $O\left(k^{2}\right)$. For constant $k$ such a cutting can be constructed in linear time [8].

Let $S(\sigma)$ be the set of segments defining $\mathcal{A}(\Pi)$ inside $\sigma$. We construct a (1/4)-cutting $\Xi(S(\sigma))$. This cutting consists of $O(1)$ triangles, each intersected by $|S(\sigma)| / 4$ segments. The idea is to find a triangle in $\Xi(S(\sigma))$ that contains a goal placement, and to proceed recursively inside that triangle. (Actually, we will recurse in two triangles.) To decide in which triangle to recurse we proceed as follows.

Let $L=\left\{\ell_{1}, \ldots, \ell_{a}\right\}$ be the set of lines through the edges of the cutting $\Xi(S(\sigma))$. On each line $\ell_{i}$ we compute the maximum overlap $\xi_{i}=\max _{r \in \ell_{i}} \omega(r)$ in $O(n+m)$ time using the algorithm of Lemma 3.3. Let's assume for the moment that all the maxima are distinct. Let $i^{*}$ be such that $\xi_{i^{*}}=\max _{i} \xi_{i}$. By Lemma 3.4 we know for each line $\ell_{i}$ with $i \neq i^{*}$ to which side we can restrict our attention. This implies that we can restrict our attention to at most two triangles (separated by the line $\left.\ell_{i^{*}}\right)$. The number of segments on which we must recurse is thus at most $|S(\sigma)| / 2$. After $O(\log (n+m))$ recursive calls we are left with two triangular regions that are not intersected by any of the segments of $\mathcal{A}(\Pi)$. Inside each of these regions, the overlap function is a second-degree polynomial, and can be computed in linear time. Once we have the polynomial we can compute its maximum in constant time, giving us the desired goal placement. The total running time for the second stage is $O((n+m) \log (n+m))$. This almost finishes the description of the algorithm. It only remains to get rid of the assumption that all maxima $\xi_{i}$ are distinct; this is done as follows.

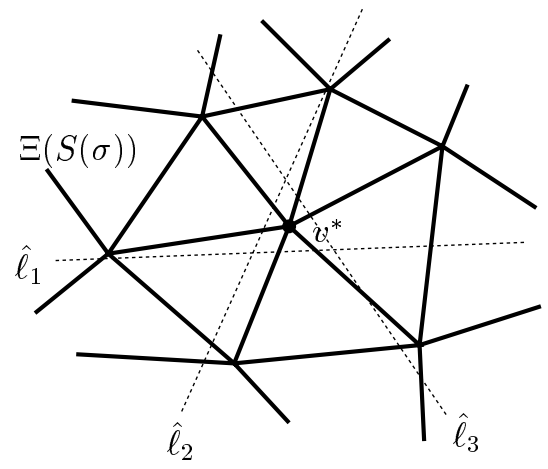

Figure 3: Dealing with a degenerate situation.

Let $\xi^{*}=\max _{i} \xi_{i}$. The difficulty arises when $\xi^{*}$ is achieved at a vertex $v^{*}$ of the cutting $\Xi(S(\sigma))$, as in Fig. 3. In this case there must be a goal position in one of the triangles of the cutting incident to $v^{*}$, but we do not know which one yet. If $v^{*}$ itself is a goal position then it doesn't matter where we recurse (provided we keep track of the the placement with the largest overlap found so far), so let's assume that this is not the case. Now, to find a triangle containing a goal placement we take three lines $\hat{\ell}_{1}, \hat{\ell}_{2}$, and $\hat{\ell}_{3}$ such that $v^{*}$ lies in the triangle $\Delta\left(\hat{\ell}_{1}, \hat{\ell}_{2}, \hat{\ell}_{3}\right)$ enclosed by them-see Fig. 3 . The distance $\delta$ from $v^{*}$ to each of the three lines should be such that there is no goal placement inside $\Delta$. This can be achieved by computing with $\delta$ symbolically, treating it as an extension of the reals which is larger than zero but smaller than any positive real. Let $\hat{\xi}_{j}:=\max _{r \in \hat{\ell}_{j}} \omega(r)$. We compute $\hat{\xi}_{j}$, for $j=1,2,3$, using Lemma 3.3 Let $\hat{r}_{j}$ be such that $\omega\left(\hat{r}_{j}\right)=\hat{\xi}_{j}$.

Lemma 3.7 If $v^{*}$ itself is not a goal position, then $\hat{\xi}_{j}>\xi^{*}$ for at least one $j \in\{1,2,3\}$. Moreover, for such a $j$ the triangle of $\Xi(S(\sigma))$ incident to $v^{*}$ containing $\hat{r}_{j}$ must contain a goal placement.

Proof: Follows from Lemma 3.4. 
Thus we can also find out where to recurse in $O((n+m) \log (n+m))$ time in degenerate cases. This completes the proof of our main result, which is summarized in the following theorem.

Theorem 3.8 Let $P$ be a convex polygon in the plane with $n$ vertices, and let $Q$ be a convex polygon with $m$ vertices. Then a placement of $Q$ that maximizes the area of $P \cap Q$ can be computed in $O((n+m) \log (n+m))$ time.

\section{Bounds on the overlap for a particular translation}

We prove in this section that we can approximate the area of the largest possible overlap by simply looking at the placement where the centroids of $P$ and $Q$ coincide. We prove that the overlap in that placement is at least $9 / 25$ of the maximum possible overlap. We also give an upper bound example where the ratio is $4 / 9$.

\subsection{Lower bound}

Let's first define some notations. The centroid of $P$ is denoted by $c_{P}$.

$$
c_{P}=\int_{u \in P} u d u / \int_{u \in P} d u=\int_{u \in P} u d u / \operatorname{area}(P)
$$

Similarly $c_{Q}$ denotes the centroid of $Q$.

In this section we will choose the origin 0 so that the overlap function is maximal at the origin, that is the reference position $Q(0)$ for $Q$ is a maximal overlap position. The maximal overlap area is thus denoted $\omega(0)$. In the sequel, we will use the polar coordinates $(r, \theta)$ with respect to that origin, and the horizontal direction; The point with polar coordinates $(1, \theta)$ will be denoted as $e_{\theta}$.

We denote by $\Omega$ the three-dimensional object bounded above by the graph of $\omega$ and below by the horizontal plane $z=0$.

$$
\Omega=\left\{(x, y, z) \in \mathbb{R}^{3}: z=\omega(x, y)\right\}
$$

Lemma 4.1 The translation $r$ that superimposes the centroids of $P$ and $Q(r)$ is given by the projection of the centroid of $\Omega$ onto $\mathbb{R}^{2}$.

Proof: The horizontal projection $p\left(c_{\Omega}\right)$ of the centroid $c_{\Omega}$ of $\Omega$ is

$$
p\left(c_{\Omega}\right)=\int_{v \in \mathbb{R}^{2}} \omega(v) v d v / \int_{v \in \mathbb{R}^{2}} \omega(v) d v
$$

replacing $\omega$ by its expression by integrals, we get

$$
\begin{aligned}
p\left(c_{\Omega}\right) & = \\
& =\int_{v \in \mathbb{R}^{2}} \int_{u \in P \cap(Q+v)} d u v d v / \int_{v \in \mathbb{R}^{2}} \int_{u \in P \cap(Q+v)} d u d v \\
& =\int_{u \in P} \int_{v \in u-Q} v d v d u / \iint_{u \in P} d v d u \\
& =\int_{u \in P \in \operatorname{Q}} \int_{v \in Q}-(u-v) d v d u / \int_{u \in P} \int_{v \in Q}-d v d u
\end{aligned}
$$


then, using the definitions of $c_{P}$ and $c_{Q}$, we obtain

$$
\begin{aligned}
p\left(c_{\Omega}\right) & = \\
& =\int_{u \in P}\left(-u+c_{Q}\right) \operatorname{area}(Q) d u / \int_{u \in P}-\operatorname{area}(Q) d u \\
& \left.=\left(c_{Q} \operatorname{area}(P) \operatorname{area}(Q)\right)+\operatorname{area}(Q) \int_{u \in P}-u d u\right) \\
& \times \frac{1}{-\operatorname{area}(P) \operatorname{area}(Q)} \\
& c_{P}-c_{Q}
\end{aligned}
$$

$p\left(c_{\Omega}\right)$ can now be evaluated in polar coordinates:

$$
\begin{aligned}
p\left(c_{\Omega}\right) & = \\
& =\int_{v \in \mathbb{R}^{2}} \omega(v) v d v / \int_{v \in \mathbb{R}^{2}} \omega(v) d v \\
& =\int_{\theta=0}^{2 \pi} \int_{r=0}^{\infty} \omega\left(r e_{\theta}\right) r e_{\theta} r d r d \theta / \int_{\theta=0}^{2 \pi} \int_{r=0}^{\infty} \omega\left(r e_{\theta}\right) r d r d \theta \\
& =\int_{\theta=0}^{2 \pi} \int_{r=0}^{\infty} \omega\left(r e_{\theta}\right) r^{2} d r e_{\theta} d \theta / \int_{\theta=0}^{2 \pi} \int_{r=0}^{\infty} \omega\left(r e_{\theta}\right) r d r e_{\theta} d \theta \\
& =\int_{\theta=0}^{2 \pi} e_{\theta} \rho(\theta) A(\theta) d \theta / \int_{\theta=0}^{2 \pi} e_{\theta} A(\theta) d \theta,
\end{aligned}
$$

where $A(\theta)=\int_{r=0}^{\infty} \omega\left(r e_{\theta}\right) r d r$ is defined as the area of the intersection of $\Omega$ with a vertical half plane with polar coordinates $(r, \theta), r \geqslant 0$. The polar coordinates of the horizontal projection of the centroid of that cross section are $(\rho(\theta), \theta)$ where $\rho(\theta)=\frac{1}{A(\theta)} \int_{r=0}^{\infty} \omega\left(r e_{\theta}\right) r^{2} d r$

Lemma 4.2 The value of $\omega$ at the projection centroid of a cross section of $\Omega$ is greater than $9 / 25$ times the maximum of $\omega$. That is $\omega\left(\rho(\theta) e_{\theta}\right) \geqslant(9 / 25) \omega(0)$, for any $\theta$.

Proof: Since $\omega\left(r e_{\theta}\right)$ is strictly decreasing from its maximum to 0 (Theorem 3.2), there is a unique value $r_{\theta}$ such that $\omega\left(r_{\theta} e_{\theta}\right)=\frac{9}{25} \omega(0)$. Now we consider the function $\omega_{\theta}^{\prime}(r)=\omega(0)\left(1-\frac{2 r}{5 r_{\theta}}\right)^{2}$ for $r \in\left[0, \frac{5}{2} r_{\theta}\right]$.

Using the downwards concavity of the function $\sqrt{\omega}$ (Theorem 3.1), the relative position of $\omega_{\theta}^{\prime}$ and $\omega$ are the following (see Figure 4):

$$
\begin{aligned}
& \omega(0)=\omega_{\theta}^{\prime}(0) \\
& \omega\left(r e_{\theta}\right) \geqslant \omega_{\theta}^{\prime}(r) \quad, \quad r \in\left[0, r_{\theta}\right] \\
& \omega\left(r e_{\theta}\right) \leqslant \omega_{\theta}^{\prime}(r) \quad, \quad r \in\left[r_{\theta}, \frac{5}{2} r_{\theta}\right] \\
& \omega\left(r e_{\theta}\right)=0 \quad, \quad r \in\left[\frac{5}{2} r_{\theta}, \infty\right]
\end{aligned}
$$


Since the weighted barycenter of function $\omega_{\theta}^{\prime}$ is

$$
\int_{r=0}^{\frac{5}{2} r_{\theta}} \omega_{\theta}^{\prime}(r) r^{2} d r / \int_{r=0}^{\frac{5}{2} r_{\theta}} \omega_{\theta}^{\prime}(r) r d r=r_{\theta}
$$

from inequalities above, we deduce that $\rho(\theta) \leqslant r_{\theta}$ and thus by Theorem 3.2: $\omega\left(\rho(\theta) e_{\theta}\right) \geqslant \omega\left(r_{\theta}\right)=$ $\frac{9}{25} \omega(0)$.

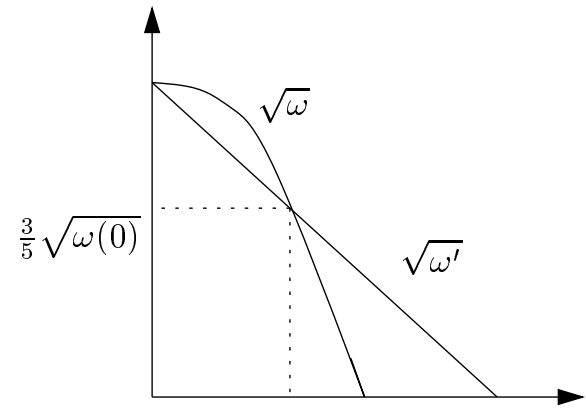

$r_{\theta}$

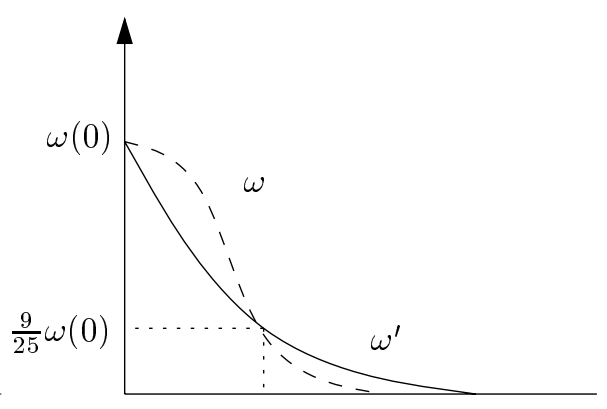

$r_{\theta}$

Figure 4: Relative position of $\omega$ and $\omega^{\prime}$

Lemma 4.3 The curve $\left(r_{\theta} e_{\theta}\right)_{0 \leqslant \theta<2 \pi}$ is convex.

Proof: It follows directly from the downwards concavity of function $\sqrt{\omega}$ (Theorem 3.1). The curve $\theta \mapsto r_{\theta} e_{\theta}$ is the intersection of the 3D surface defined by $z=\sqrt{\omega}$ and the horizontal plane $z=\frac{3}{5} \sqrt{\omega(0)}$.

Theorem 4.4 The translation which matches the centroids of two convex polygons realizes an overlap area of at least $9 / 25$ of the maximal overlap area.

Proof: The overlap at the placement where the centroids of $P$ and $Q$ coincide is $\omega\left(c_{Q}-c_{P}\right)$, which is $\omega\left(p\left(c_{\Omega}\right)\right)$ by Lemma 4.1. $p\left(c_{\Omega}\right)$ is the centroid of points $\rho(\theta) e_{\theta}$ weighted by the positive function $A(\theta)$ (Equation 1). The curve $\rho(\theta) e_{\theta}$ is inside the convex curve $r_{\theta} e_{\theta}$ (using Lemma 4.2), which is convex by Lemma 4.3. Thus $p\left(c_{\Omega}\right)$ is inside the convex curve $r_{\theta} e_{\theta}$ and thus, by the downwards concavity of $\sqrt{\omega}$ (Theorem 3.1), $\omega\left(p\left(c_{\Omega}\right)\right) \geqslant \omega\left(r_{\theta} e_{\theta}\right)=\frac{9}{25} \omega(0)$.

Theorem 4.5 The translation which matches the centroids of two $d$-dimensional convex polyhedra realizes an overlap volume of at least $(3 /(d+3))^{d}$ times the maximal overlap volume.

Proof: The proof is similar to the one we gave for the two dimensional case. In higher dimension we use the downwards concavity of the function $\sqrt[d]{\omega}$ (which follows again from the BrunnMinkowski Theorem); and the new definition of $\omega^{\prime}(r)$ is $\omega(0)\left(1-\frac{2 r}{(d+3) r_{\theta}}\right)^{d}$ which yields the claimed bound. 


\subsection{Upper bounds}

\subsubsection{Two dimensional example}

The worst known example for overlapping two polygons reaches a bound of $4 / 9$ between the maximal overlap and the overlap at the centroid position. The example is depicted in Figure 5, assuming that the small edges of triangles have length 1 , and that the opposite angle is very small. The intersection at the optimal position is a small square of edge length about 1 , and the intersection at the centroid position is a square of edge length about $2 / 3$.

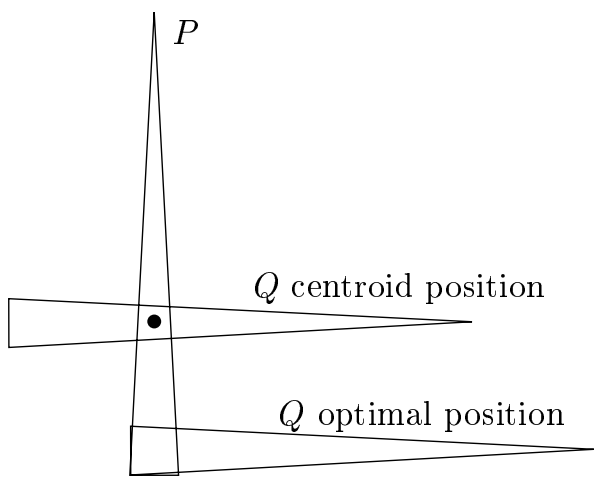

Figure 5: Example reaching $\frac{4}{9}$ upper bound.

\subsubsection{Three dimensional example}

Our worst example in three dimensions is drawn in Figure 6. If edge $e$ has length 1 , and the smallest face of $Q$ as area $\varepsilon$ then the volume of the intersection at the optimal position is $\varepsilon$. Let's now evaluate the volume when the centroids coincide: edge $e^{\prime}$ parallel to $e$ in the section of $P$ by the vertical plane through the centroid of $P$ has length $3 / 4$, the length of the stick $Q$ inside $P$ is about $2 / 3$ of $e^{\prime}$, that is $1 / 2$, and finally the horizontal section of $Q$ at its centroid is a $3 / 4$ homothet of the horizontal face of $Q$. Thus the volume of the intersection is $\frac{1}{2}\left(\frac{3}{4}\right)^{2} \varepsilon=\frac{9}{32} \varepsilon$.

\section{Conclusions}

We presented an algorithm that computes a translation of a convex polygon $Q$ that maximizes the area of overlap with another convex polygon $P$. The algorithm runs in $O((n+m) \log (n+m))$ time, where $n$ and $m$ are the number of vertices of $P$ and $Q$, respectively. Our algorithm is based on the unimodality of the overlap function for convex polygons. We showed that the particular placement where the centroids of the polygons coincide gives an approximation of the optimal overlap area of at least $9 / 25$. We conjecture that the lower bound can be improved to $4 / 9$, which would be a tight bound, as we have shown. The demonstration of any such better bound, however, would have to rely on some other facts than Theorem 3.1. In fact, it is easy to construct an example of a bivariate function $\delta$ such that $\sqrt{\delta}$ is downwards concave and the value of $\delta$ at its centroid is only $\frac{9}{25}$ of its maximal value, but such $\delta$ does not seem to be the representation of the $\omega$ function related to the overlap of two polygons.

The obvious next step is to develop an efficient algorithm for arbitrary simple polygons. Unfortunately, the overlap is no longer unimodal for non-convex polygons: the overlap function can have up to $\Theta\left(n^{2} m^{2}\right)$ local maxima. It seems difficult to develop an algorithm that does not inspect 


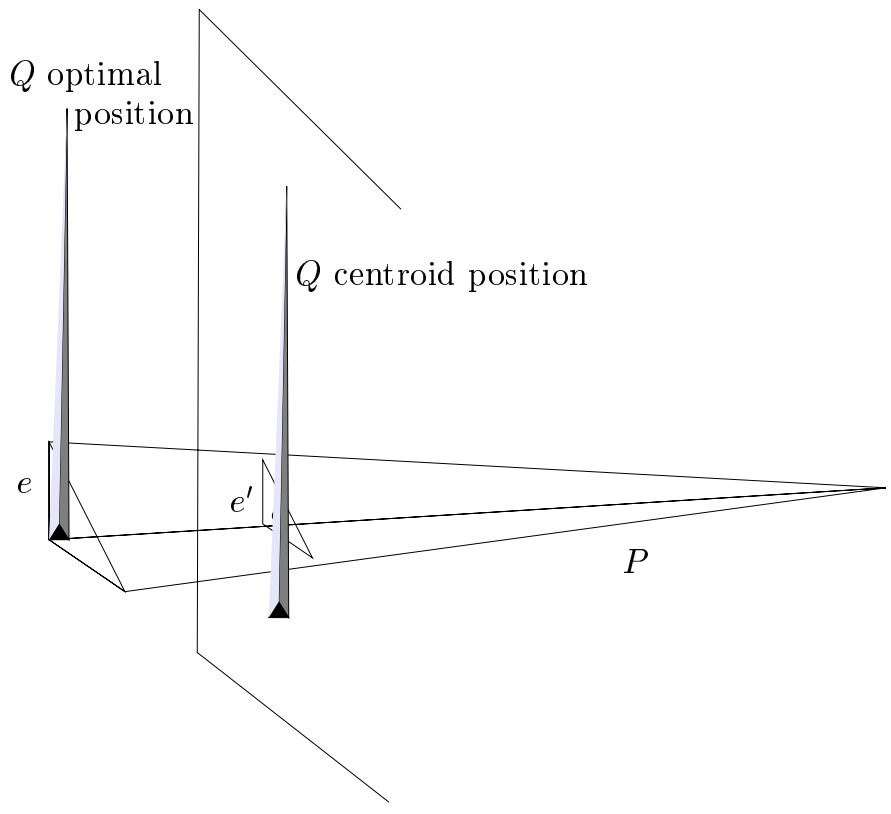

Figure 6: Example reaching $\frac{9}{32}$ upper bound.

all local maxima. In many cases, however, the number of local maxima in the overlap function is relatively small. It would be interesting to develop an algorithm whose running time depends on the number of local maxima.

Acknowledgment. Thanks to H. Alt for helpful discussions about the centroid problem.

\section{References}

[1] Pankaj K. Agarwal, Micha Sharir, and Sivan Toledo. Applications of parametric searching in geometric optimization. In Proc. 3rd ACM-SIAM Sympos. Discrete Algorithms, pages 72-82, 1992.

[2] H. Alt, B. Behrends, and J. Blömer. Approximate matching of polygonal shapes. In Proc. 7th Annu. ACM Sympos. Comput. Geom., pages 186-193, 1991.

[3] H. Alt and M. Godau. Measuring the resemblance of polygonal curves. In Proc. 8th Annu. ACM Sympos. Comput. Geom., pages 102-109, 1992.

[4] D. Avis, P. Bose, T. Shermer, J. Snoeyink, G. Toussaint, and B. Zhu. On the sectional area of convex polytopes. In Communication at the 12th Annu. ACM Sympos. Comput. Geom., page C, 1996.

[5] F. Avnaim and J.-D. Boissonnat. Polygon placement under translation and rotation. In Proc. 5th Sympos. Theoret. Aspects Comput. Sci., volume 294 of Lecture Notes in Computer Science, pages 322-333. Springer-Verlag, 1988.

[6] B. Chazelle. The polygon containment problem. In F. P. Preparata, editor, Computational Geometry, volume 1 of Advances in Computing Research, pages 1-33. JAI Press, London, England, 1983. 
[7] B. Chazelle. An optimal algorithm for intersecting three-dimensional convex polyhedra. SIAM J. Comput., 21(4):671-696, 1992.

[8] B. Chazelle. Cutting hyperplanes for divide-and-conquer. Discrete Comput. Geom., 9(2):145$158,1993$.

[9] L. P. Chew, M. T. Goodrich, D. P. Huttenlocher, K. Kedem, J. M. Kleinberg, and D. Kravets. Geometric pattern matching under Euclidean motion. In Proc. 5th Canad. Conf. Comput. Geom., pages 151-156, Waterloo, Canada, 1993.

[10] L. P. Chew and K. Kedem. Placing the largest similar copy of a convex polygon among polygonal obstacles. Comput. Geom. Theory Appl., 3(2):59-89, 1993.

[11] S. J. Fortune. A fast algorithm for polygon containment by translation. In Proc. 12th Internat. Colloq. Automata Lang. Program., volume 194 of Lecture Notes in Computer Science, pages 189-198. Springer-Verlag, 1985.

[12] G. N. Frederickson and D. B. Johnson. Generalized selection and ranking: sorted matrices. SIAM J. Comput., 13:14-30, 1984.

[13] B. Grünbaum. Convex Polytopes. Wiley, New York, NY, 1967.

[14] D. Halperin. Algorithmic Motion Planning via Arrangements of Curves and of Surfaces. Ph.D. thesis, Computer Science Department, Tel-Aviv University, Tel Aviv, 1992.

[15] D. P. Huttenlocher, K. Kedem, and J. M. Kleinberg. On dynamic Voronoi diagrams and the minimum Hausdorff distance for point sets under Euclidean motion in the plane. In Proc. 8th Annu. ACM Sympos. Comput. Geom., pages 110-120, 1992.

[16] D. P. Huttenlocher, K. Kedem, and M. Sharir. The upper envelope of Voronoi surfaces and its applications. Discrete Comput. Geom., 9:267-291, 1993.

[17] J.-C. Latombe. Robot Motion Planning. Kluwer Academic Publishers, Boston, 1991.

[18] D.M. Mount, R. Silverman, and A. Wu. On the area of overlap of translated polygons. SPIE Vision Geometry II, 2060:254-264, 1993.

[19] D. Parson and C. Torras. The combinatorics of overlapping convex polygons in contact. In Proc. 4th Canad. Conf. Comput. Geom., pages 83-92, 1992.

[20] M. Sharir, R. Cole, K. Kedem, D. Leven, R. Pollack, and S. Sifrony. Geometric applications of Davenport-Schinzel sequences. In Proc. 27th Annu. IEEE Sympos. Found. Comput. Sci., pages $77-86,1986$.

[21] R. Venkatasubramanian. On the area of intersection of two closed 2D objects. Information Sciences, 82:25-44, 1995. 